PROCEEDINGS OF THE

AMERICAN MATHEMATICAL SOCIETY

Volume 131, Number 11, Pages 3407-3410

S 0002-9939(03)07176-4

Article electronically published on June 19, 2003

\title{
BANACH SPACES HAVING THE RADON-NIKODỲM PROPERTY AND NUMERICAL INDEX 1
}

\author{
MIGUEL MARTÍN
}

(Communicated by Jonathan M. Borwein)

\begin{abstract}
Let $X$ be a Banach space with the Radon-Nikodỳm property. Then, the following are equivalent.

(i) $X$ has numerical index 1 .

(ii) $\left|x^{* *}\left(x^{*}\right)\right|=1$ for all $x^{*} \in \operatorname{ex}\left(B_{X^{*}}\right)$ and $x^{* *} \in \operatorname{ex}\left(B_{X^{* *}}\right)$.

(iii) $X$ is an almost-CL-space.

(iv) There are a compact Hausdorff space $K$ and a linear isometry $J: X \rightarrow$ $C(K)$ such that $\left|x^{* *}\left(J^{*} \delta_{s}\right)\right|=1$ for all $s \in K$ and $x^{* *} \in \operatorname{ex}\left(B_{X^{* *}}\right)$.

If $X$ is a real space, the above conditions are equivalent to being semi-nicely embedded in some space $C(K)$.
\end{abstract}

The numerical index of a Banach space is a constant relating the norm and the numerical radius of operators on the space. Let us present the relevant definitions. For a Banach space $X$, we write $B_{X}$ for the closed unit ball and $S_{X}$ for the unit sphere. We denote by $X^{*}$ the dual space and by $L(X)$ the Banach algebra of all bounded linear operators on $X$. For such an operator $T$, the numerical radius of $T$ is

$$
v(T)=\sup \left\{\left|x^{*}(T x)\right|: x^{*} \in S_{X^{*}}, x \in S_{X}, x^{*}(x)=1\right\} .
$$

The numerical index of the space $X$ is then given by

$$
n(X)=\max \{k \geq 0: k\|T\| \leq v(T) \quad \forall T \in L(X)\} .
$$

We refer the reader to the books [3, 4] and to the expository paper [13] for general information and background. Recent results can be found in [7, 12, 14, 15].

Let us mention here some facts concerning the numerical index which will be relevant to our discussion. First, one has $v\left(T^{*}\right)=v(T)$ for every $T \in L(X)$, where $T^{*}$ is the adjoint operator of $T$ (see $[3, \S 9]$ ), and it clearly follows that $n\left(X^{*}\right) \leq n(X)$. The question whether this is actually an equality seems to be open. Second, it is a classical result that $L$ - and $M$-spaces have numerical index 1 [6].

The aim of this paper is to characterize Banach spaces with numerical index 1 among those having the Radon-Nikodỳm property (RNP for short; see [5] for background). To this end, we prove that some general sufficient conditions for a Banach space to have numerical index 1 are also necessary if the space has the RNP.

Received by the editors November 20, 2001.

2000 Mathematics Subject Classification. Primary 46B20, 47A12.

This research was partially supported by Spanish MCYT projects no. BFM2000-1467 and BFM2002-00061. 
Let us introduce some definitions and comments. A Banach space $X$ is an almost$C L$-space if $B_{X}$ is the absolutely closed convex hull of every maximal convex subset of $S_{X}$. This notion was introduced by $\AA$. Lima [10], generalizing the concept of CLspace given by R. Fullerton [8] in 1960. Real and complex almost-CL-spaces have numerical index 1 (see [13, §4] or [1]), but it is not known whether the reciprocal result is true. As usual, we write $C(K)$ for the Banach space of all continuous functions from the compact Hausdorff space $K$ into the scalar field. Given $s \in K$, $\delta_{s}$ stands for the functional $f \longmapsto f(s)$ on $C(K)$. Finally, we write $\operatorname{co}(B)$ for the convex-hull of $B$ and, given a convex subset $A$ of $X, \operatorname{ex}(A)$ and $\operatorname{dent}(A)$ are, respectively, the set of extreme points and the set of denting points of $A$.

The main result of the paper is the following.

Theorem 1. Let $X$ be a Banach space having the RNP. Then the following are equivalent.

(i) $n(X)=1$

(ii) $\left|x^{* *}\left(x^{*}\right)\right|=1$ for all $x^{*} \in \operatorname{ex}\left(B_{X^{*}}\right)$ and $x^{* *} \in \operatorname{ex}\left(B_{X^{* *}}\right)$.

(iii) $X$ is an almost-CL-space.

(iv) There are a compact Hausdorff space $K$ and a linear isometry $J: X \rightarrow$ $C(K)$ such that $\left|x^{* *}\left(J^{*} \delta_{s}\right)\right|=1$ for all $s \in K$ and $x^{* *} \in \operatorname{ex}\left(B_{X^{* *}}\right)$.

Proof. The implication $(i i) \Rightarrow(i)$ is straightforward. Just use that $v(T)=v\left(T^{*}\right)$ for every $T \in L(X)$. The implication $(i i i) \Rightarrow(i)$ has been discussed above.

$(i v) \Rightarrow(i)$. We fix $T \in L(X)$ and we have to prove that $v(T)=\|T\|$. For each $s \in K$, we take $x^{* *} \in \operatorname{ex}\left(B_{X^{* *}}\right)$ such that

$$
\left|x^{* *}\left(T^{*}\left(J^{*} \delta_{s}\right)\right)\right|=\left\|T^{*}\left(J^{*} \delta_{s}\right)\right\| .
$$

Since $\left|x^{* *}\left(J^{*} \delta_{s}\right)\right|=1$, we have

$$
v(T)=v\left(T^{*}\right) \geq\left\|T^{*}\left(J^{*} \delta_{s}\right)\right\|
$$

for each $s \in K$. It is clear that

$$
\|T\|=\left\|T^{*}\right\|=\sup \left\{\left\|T^{*}\left(J^{*} \delta_{s}\right)\right\|: s \in K\right\}
$$

and, so, $v(T)=\|T\|$ and $n(X)=1$.

$(i) \Rightarrow(i i)$. We use [12, Lemma 1] to get $\left|x^{*}(x)\right|=1$ for all $x \in \operatorname{dent}\left(B_{X}\right)$ and $x^{*} \in \operatorname{ex}\left(B_{X^{*}}\right)$. Since $X$ has the RNP, it may be concluded that

$$
\operatorname{ex}\left(B_{X^{* *}}\right) \subseteq \overline{\operatorname{dent}\left(B_{X}\right)} w^{*},
$$

so $\left|x^{* *}\left(x^{*}\right)\right|=1$ for all $x^{*} \in \operatorname{ex}\left(B_{X^{*}}\right)$ and $x^{* *} \in \operatorname{ex}\left(B_{X^{* *}}\right)$.

$(i) \Rightarrow($ iii $)$. Let $F$ be a maximal convex subset of $S_{X}$. By using Hahn-Banach and Krein-Milman Theorems, it is easily checked that there exists $x^{*} \in \operatorname{ex}\left(B_{X^{*}}\right)$ such that

$$
F=\left\{x \in B_{X}: x^{*}(x)=1\right\} .
$$

So, by [12, Lemma 1], $\operatorname{dent}\left(B_{X}\right)$ is contained in the absolutely convex hull of $F$. On the other hand, since $X$ has the RNP, $B_{X}=\overline{\operatorname{co}}\left(\operatorname{dent}\left(B_{X}\right)\right)$. Thus, $B_{X}$ is the closed absolutely convex hull of $F$ and $X$ is an almost-CL-space.

$(i) \Rightarrow(i v)$. For $x^{*} \in B_{X^{*}}$, we have

$$
x^{*} \in \operatorname{ex}\left(B_{X^{*}}\right) \Leftrightarrow\left|x^{*}(x)\right|=1 \text { for each } x \in \operatorname{dent}\left(B_{X}\right) .
$$

One implication is [12, Lemma 1]; the other one arises from the fact that dent $\left(B_{X}\right)$ is norming for $X^{*}$. 
It follows from (11) that $K=\operatorname{ex}\left(B_{X^{*}}\right)$, with the $w^{*}$ topology, is a Hausdorff compact space. Let $J$ be the canonical injection from $X$ into $C(K)$. Now, since $J^{*} \delta_{s}=s \in \operatorname{ex}\left(B_{X^{*}}\right)$, we deduce from $(i i)$ that $\left|x^{* *}\left(J^{*} \delta_{s}\right)\right|=1$ for all $s \in K$ and $x^{* *} \in \operatorname{ex}\left(B_{X^{* *}}\right)$.

Let us give some remarks on the above theorem.

Condition (ii) of the theorem is called E.P.I.P. in [10] and appeared in [11, §4]. In [10. Corollary 3.6], Å. Lima proved $(i i) \Rightarrow(i i i)$ for arbitrary real Banach spaces. Nevertheless, we do not know if Lima's result is valid in the complex case without the RNP assumption.

Observe that, when proving $(i v) \Rightarrow(i)$, we do not need the RNP and that the result still holds if we change $C(K)$ to $C_{b}(\Omega)$, the Banach space of all bounded continuous functions from the topological Hausdorff space $\Omega$ into the scalar field. Therefore, we have the following by-product of the proof of Theorem 1

Corollary 2. Let $\Omega$ be a Hausdorff topological space and let $X$ be a Banach space. If there exists a linear isometry $J: X \rightarrow C_{b}(\Omega)$ such that

$$
\left|x^{* *}\left(J^{*} \delta_{s}\right)\right|=1 \quad\left(s \in \Omega, x^{* *} \in \operatorname{ex}\left(B_{X^{* *}}\right)\right),
$$

then $n(X)=1$.

The above corollary improves one result recently given by D. Werner 16, Corollary 2.2]. Some definitions are required. Following [16], a Banach space $X$ is said to be nicely embedded in $C_{b}(\Omega)$ if there exists a linear isometry $J: X \rightarrow C_{b}(\Omega)$ such that for all $s \in \Omega$ the following properties are satisfied:

(N1) $\left\|J^{*} \delta_{s}\right\|=1$.

(N2) $\operatorname{span}\left(J^{*} \delta_{s}\right)$ is an $L$-summand in $X^{*}$.

It is clear that nicely embedded spaces fulfill the conditions in Corollary [2, so they have numerical index 1. This is precisely Corollary 2.2 of [16] (see also the introduction of [14]). But the converse result is false even in the finite-dimensional setting. As a matter of fact, in the case when $X$ is the 3 -dimensional $L$-space, $n(X)=1$ and $X^{*}$ does not have any non-trivial $L$-summand.

In the real case, Corollary 2 can be written in a more suitable form by using notation similar to Werner's result. Following Å. Lima [9, 10, a closed subspace $Y$ of a Banach space $X$ is said to be a semi $L$-summand if for every $x \in X$ there exists a unique $y \in Y$ such that $\|x-y\|=d(x, Y)$, and moreover this $y$ satisfies $\|x\|=\|y\|+\|x-y\|$. A Banach space $X$ is said to be semi-nicely embedded in $C_{b}(\Omega)$ if there exists a linear isometry $J: X \rightarrow C_{b}(\Omega)$ such that for all $s \in \Omega$ the following properties are satisfied:

(N1) $\left\|J^{*} \delta_{s}\right\|=1$.

$\left(\mathrm{N} 2^{\prime}\right) \operatorname{span}\left(J^{*} \delta_{s}\right)$ is a semi $L$-summand in $X^{*}$.

Given a real Banach space $X$ and a point $x \in S_{X}$, [10] Theorem 3.1] says that $\operatorname{span}(x)$ is a semi- $L$-summand if and only if $\left|x^{*}(x)\right|=1$ for all $x^{*} \in \operatorname{ex}\left(B_{X^{*}}\right)$. Therefore, condition $(i v)$ in Theorem 1 is equivalent to the property of being seminicely embedded in some $C(K)$. Therefore, for real spaces, Theorem 1 reads as follows.

Corollary 3. Let $X$ be a real Banach space having the $R N P$. Then $n(X)=1$ if and only if $X$ is semi-nicely embedded in some $C(K)$. 
It is now easy to give examples of Banach spaces semi-nicely embedded in some $C(K)$ which are not nicely embedded in any $C_{b}(\Omega)$. For instance, since the real space $l_{1}$ has the RNP and $n\left(l_{1}\right)=1$, it is semi-nicely embedded in $C(\Delta)$, where $\Delta$ is the Cantor set. But $l_{\infty}$ does not have any non-trivial $L$-summand (see 2 . Theorem 6.15], for instance).

\section{REFERENCES}

[1] M. D. Acosta, CL-spaces and numerical radius attaining operators, Extracta Math. 5 (1990), $138-140$.

[2] E. Behrends, M-structure and the Banach-Stone Theorem, Lecture Notes in Math. 736, Springer-Verlag, Berlin, 1979. MR 81b:46002

[3] F. F. Bonsall and J. Duncan, "Numerical Ranges of Operators on Normed Spaces and of Elements of Normed Algebras", London Math. Soc. Lecture Note Ser. 2, Cambridge University Press, 1971. MR 44:5779

[4] F. F. Bonsall and J. Duncan, "Numerical Ranges II", London Math. Soc. Lecture Note Ser. 10, Cambridge University Press, 1973. MR 56:1063

[5] J. Diestel and J. J. Uhl, "Vector Measures", Math. Surveys 15, Amer. Math. Soc., Providence, RI, 1977. MR 56:12216

[6] J. Duncan, C. M. McGregor, J. D. Pryce, and A. J. White, The numerical index of a normed space, J. London Math. Soc. 2 (1970), 481-488. MR 41:8967

[7] C. Finet, M. Martín, and R. Payá, Numerical index and renorming, Proc. Amer. Math. Soc. 131 (2003), 871-877.

[8] R. E. Fullerton, Geometrical characterizations of certain function spaces. In: Proc. Internat. Sympos. Linear Spaces (Jerusalem, 1960), pp. 227-236. Pergamon, Oxford, 1961. MR 24:A2834

[9] Å. Lima, Intersection properties of balls and subspaces in Banach spaces, Trans. Amer. Math. Soc. 227 (1977), 1-62. MR 55:3752

[10] Å. Lima, Intersection properties of balls in spaces of compact operators, Ann. Inst. Fourier, Grenoble 28 (1978), 35-65. MR 80g:47048

[11] J. Lindenstrauss, Extension of compact operators, Memoirs Amer. Math. Soc. 48, Providence, RI, 1964. MR 31:3828

[12] G. López, M. Martín, and R. Payá, Real Banach spaces with numerical index 1, Bull. London Math. Soc. 31 (1999), 207-212. MR 99k:46024

[13] M. Martín, A survey on the numerical index of a Banach space, Extracta Math. 15 (2000), 265-276. MR 2002b:46027

[14] M. Martín and R. Payá, Numerical index of vector-valued function spaces, Studia Math. 142 (2000), 269-280. MR 2001i:46017

[15] M. Martín and A. R. Villena, Numerical index and Daugavet property for $L_{\infty}(\mu, X)$, to appear in Proc. Edinburgh Math. Soc.

[16] D. Werner, The Daugavet equation for operators on function spaces, J. Funct. Anal. 143 (1997), 117-128. MR 98c:47025

Departamento de Análisis Matemático, Facultad de Ciencias, Universidad de Granada, 18071 Granada, Spain

E-mail address: mmartins@ugr.es 